\title{
Malika ZEGHAL, (dir.), Intellectuels de l'islam contemporain. Nouvelles générations, nouveaux débats
}

Revue des mondes musulmans et de la Méditerranée, 123, 2008, pp. 32-201.

\section{Hartmut Fähndrich}

\section{CpenEdition} Journals

Édition électronique

URL : http://journals.openedition.org/assr/21689

DOI : 10.4000/assr.21689

ISSN : $1777-5825$

Éditeur

Éditions de l'EHESS

Édition imprimée

Date de publication : 31 décembre 2009

Pagination : 75-342

ISBN : 978-2-7132-2218-4

ISSN : 0335-5985

Référence électronique

Hartmut Fähndrich, « Malika zeghaL, (dir.), Intellectuels de l'islam contemporain. Nouvelles générations, nouveaux débats ", Archives de sciences sociales des religions [En ligne], 148 | octobre-décembre 2009, document 148-134, mis en ligne le 27 janvier 2010, consulté le 21 septembre 2020. URL : http:// journals.openedition.org/assr/21689; DOI : https://doi.org/10.4000/assr.21689

Ce document a été généré automatiquement le 21 septembre 2020.

(C) Archives de sciences sociales des religions 


\section{Malika ZEGHAL, (dir.), Intellectuels de l'islam contemporain. Nouvelles générations, nouveaux débats}

Revue des mondes musulmans et de la Méditerranée, 123, 2008, pp. 32-201.

\section{Hartmut Fähndrich}

\section{RÉFÉRENCE}

Malika ZEGHAL, (dir.), Intellectuels de l'islam contemporain. Nouvelles générations, nouveaux débats, Revue des mondes musulmans et de la Méditerranée, 123, 2008, pp. 32-201.

1 La Revue des mondes musulmans et de la Méditerranée (REMMM) est certainement un des journaux les plus intéressants sur le Proche- et Moyen Orient et le monde musulman. Une partie de cette qualité est due au fait que chaque numéro est consacré à un thème particulier discuté en profondeur dans plusieurs contributions.

2 Le numéro 123 (2008-1) met l'accent sur les «Intellectuels de l'islam contemporain. Nouvelles générations, nouveaux débats ». Deux tiers des trois cent dix pages sont consacrés à ce sujet, le dernier tiers se partageant entre deux articles sur la Turquie contemporaine et des comptes rendus. Malgré le grand nombre des publications en langues européennes sur les développements des débats musulmans la collection ici réunie offre des analyses originales et des résultats fascinants, voire stupéfiants.

3 L'observation la plus intéressante, et qui se retrouve dans presque toutes les contributions, est celle de l'adieu à certaines notions cultivées depuis longtemps chez beaucoup de musulmans, en particulier celle de la confrontation de la culture/ civilisation musulmane/islamique avec « l'Ouest » y compris la critique permanente de cet Ouest considéré comme responsable de tout ce qui se passe dans le monde musulman; celle, aussi, d'un islam uniforme réunissant les croyants musulmans du 
monde entier sous un seul drapeau vert leur inspirant la même mode de penser et la même façon de vivre. S'y exprime, en revanche, de plus en plus, et non seulement parmi les communautés musulmanes dans l'Ouest, l'idée d'un monde en plein développement obligeant les religions à se développer, à s'ouvrir, à devenir pluralistes.

Les huit articles qui suivent l'introduction de Malika Zeghal, «Réformisme, islamisme et libéralisme religieux ", traitent, chacun, du développement dans un pays particulier. Sont ainsi présentés des débats musulmans en Indonésie (John Bowen : «Intellectual Pilgrimages and Local Norms in Fashioning Indonesian Islam») et au Pakistan (Muhammad Qasim Zaman: "Religious Discourse and the public Sphere in Contemporary Pakistan»), le titre de cette première section étant «Figures réformistes, productions intellectuelles et normes publiques »; l'Allemagne (Jeannette Jouili et Nikola Tietze: "L'émergence de discours intellectuels islamiques en Allemagne : entre stratégie politique et herméneutique féministe »), la France (Franck Frégosi : « Les nouveaux contours du champs intellectuel musulman en France ») et les États-Unis (Malika Zeghal: "Nouvelles contraintes discursives et réformismes religieux : Les intellectuels musulmans dans la sphère publique américaine »), le titre de cette deuxième section étant "Minorités musulmanes entre intermédiaires culturels et intellectuels libéraux»; et, l'Arabie saoudite (Stéphanie Lacroix: «Les nouveaux intellectuels religieux saoudiens: le wahhabisme en question»), la Palestine (Dyala Hamzah: "Lutte nationale ou fondation étatique? Les intellectuels palestiniens, d'Oslo I à Intifada II ») et l'Égypte (Patrick Haenni, Husam Tammam : « Penser dans l'audelà de l'islamisme: Égypte, la dispersion idéologique des lendemains qui déchantent »), le titre de cette troisième section étant "Les transformations de l'islamisme dans le monde arabe ».

$5 \quad$ En guise d'introduction, M. Zeghal présente et élabore les changements dans les cercles en cours de formation dans différents pays et communautés pour prendre le relais de ceux qui jusqu'ici se font les interprètes des musulmans. Ces nouveaux individus empruntent de nouvelles voies: ils se distancient des anciennes images de confrontation; ils ne regardent plus l'islam comme un conglomérat de rites et de normes; ils ont tendance à intérioriser l'expérience religieuse en cherchant "l'ennemi » plutôt chez ou à l'intérieur d'eux même; ils se voient, en tant que musulmans, comme participants aux valeurs universelles, non comme opposants aux valeurs des "autres»; et, finalement, ils relisent leurs textes religieux d'une façon contextualisée et historicisée. C'est-à-dire qu'ils acceptent, à l'encontre de leurs aïeux salafistes et fondamentalistes, l'histoire et la culture comme des éléments essentiels pour la compréhension de l'islam. Comme le dit M. Zeghal : «L'herméneutique devient le mot d'ordre : le contexte et l'histoire sont importants pour comprendre les textes révélés. »

Suivent les études, dont chacune traite d'un pays particuliers :

Il y a une longue tradition de voyage de l'Indonésie au "centre» arabe du monde musulman avec des conséquences importantes pour le développement de l'islam dans l'archipel, à savoir le transfert des dogmes, des traditions, des règles, ainsi que l'encouragement d'un "dialogue » entre les traditions locales et les importations du Moyen Orient. Par cette voie, les étudiants-voyageurs n'ont pas seulement introduit en Indonésie le modernisme islamique d'un Jamal al-Din al-Afghani et d'un Muhammad Abduh mais, plus tard, les œuvre des Frères musulmans et, avec l'aide financière saoudienne, l'enseignement salafiste. Pourtant, aujourd'hui comme hier, «the 
globalization of Islam ... passes by way of a continuing dialogue with local ways of life » (p. 53).

8 Au Pakistan, comme ailleurs, un des grands problèmes qui se posent dans le cadre des débats réformistes, c'est l'incompréhension mutuelle des différents groupes due aux différences dans les discours des oulémas et des nouveaux intellectuels religieux. Pour Fazlur Rahman, un des grands réformistes, d'origine pakistanaise, l'autre problème était sa proximité avec le régime d'Ayyub Khan qui, en raison de sa politique autoritaire, était très contesté. Fazlur Rahman, avant sa fuite aux États-Unis, était employé à l'Institute of Islamic Research, institution créée par le régime pour rendre compatibles les normes musulmanes avec ses initiatives modernisantes. C'est dans ce cadre que Fazlur Rahman exprimait ses doutes sur l'authenticité de certains hadîths, suggérait un rôle actif du prophète dans le processus des révélations coranique, et constatait que les normes légales du Coran n'étaient pas éternelles. Dans un débat célèbre sur la permissibilité du ribā (intérêt/usure) auquel participaient plusieurs groupes d'oulémas et de juristes, il utilisa la pensée traditionnelle pour le réfuter.

Le discours islamique en Allemagne est le résultat de l'établissement de l'islam dans ce pays qui lui-même résulte de l'histoire de l'immigration. Ce discours se développe face à «la concurrence des organisations islamiques en liens politiques avec la Turquie... et la reconnaissance statutaire dans le système institutionnel ». Les auteurs de l'article ont choisi deux tendances représentées par deux institutions : le Zentralrat der Muslime in Deutschland (ZMD) et le Zentrum für Islamische Frauenforschung und Frauenförderung (ZIF). Le ZMD a publié, en 2002, une charte Islamique comme guide pour les organisations dont il est porte-parole. Le ZIF, étant une institution pour la recherche féminine islamique et pour l'encouragement des femmes, cherche, par ses publications, à stimuler le débat à l'intérieur de la communauté musulmane. Les thématiques y sont: "l'égalité ontologique entre homme et femme, les figures féminines dans le Coran en tant que modèles pour des femmes libérées et le refus de la justification religieuse de la violence conjugale » (p.88) Avec ses publications, le ZIF veut donc participer aux débat pour promouvoir les interprétations de l'islam. En revanche, la charte du ZMD se dérobe au moindre geste d'une mise en question des normes traditionnelles islamiques. Tout ce qu'on y trouve, c'est l'encouragement des musulmans résidant en Allemagne à respecter les lois du pays. La charte sert ainsi à réunir un grand nombre de musulmans sans les obliger à analyser le rapport entre leurs propres convictions traditionnelles et les principes dominant la vie dans leur pays de résidence.

En France, le paysage intellectuel musulman est plus riche que la présence médiatique de quelques figures emblématiques ne le laisse croire, et dans cette multitude d'opinions et de tendances il y a de nouveaux porte-paroles réunies dans la tâche de " réagir à la fois aux surenchères fondamentalistes comme aux dérives islamophobes » (p. 97) suivant deux tendances différentes : soit celui des apologistes religieux, soit celui des intellectuels laïques. Les représentants des deux tendances argumentent et agissent " en vue de redonner ses lettres de noblesse à un islam perçu comme plus authentique que celui réellement pratiqué ou déclamé par ces interprètes radicaux» (p.99). La tentative de classer "six types d'opérateurs dans le champ intellectuel musulman hexagonal» (p.99), est une présentation non sans ironie bienvenue dans un domaine dominé, en général, par le sérieux: on y trouve «les médiateurs» et «les 
vulgarisateurs ", « les outsiders » et « les nouveaux talents », « les alarmistes » et « les libres contradicteurs » qui tous se partagent l'arène du discours musulman en France.

11 Après le 11 septembre 2001, est apparue, aux États-Unis, une tendance progressive et libérale s'est formée mettant en question la compréhension de l'islam liée, en général, aux mosquées, tendance accueilli très souvent avec intérêt dans le public américain, mais avec méfiance par des cercles musulmans eux-mêmes. Cette mise en question des valeurs islamiques traditionnelles ou même rétrogrades coïncidait parfois, et involontairement, avec les justifications présentées par le gouvernement Bush pour sa "guerre contre le terrorisme ». Deux individus sont choisis comme origine de la pensée des nouveaux intellectuels musulmans aux États-Unis, bien que la situation des musulmans s'y soit considérablement modifiée depuis l'époque de leur activité pendant les années soixante, soixante-dix et quatre-vingts: Isma'il al-Faruqi (1920-1986), d'origine palestinienne, et Fazlur Rahman (1919-1988), d'origine pakistanaise. Le premier a envisagé « un grand projet utopique, et cher à de nombreux islamistes de sa génération, de conceptualiser l'ensemble des sciences - sociales et naturelles - en modifiant leur contenu pour que celui-ci converge avec les enseignements de l'islam » (p. 123). Le second, en revanche, « revient à l'idée d'une lecture directe et immédiate du Coran, informée par le contexte issu de la Sunna », c'est-à-dire qu'« il formule l'idée d'une véritable réforme interprétative... une herméneutique du Coran» (p.126) à l'instar de H. G. Gadamer. Le 11 septembre a donné, après l'assimilation du grand choc, naissance à une «nouvelle minorité active... une nébuleuse de courants polyphoniques plutôt qu'une force homogène" (p.129). Pour ce mouvement composé de plusieurs voix maintenant bien écoutées aux États-Unis, "plutôt que d'islamiser la société, il s'agit donc d'humaniser la religion, qui n'existe que par la médiation humaine des individus et communautés qui lui donnent sens » (p. 132).

Depuis la fin des années quatre-vingt-dix, on observe, en Arabie Saoudite, un courant réformiste islamo-libéral dont les adhérents, beaucoup étant wahhabites, invitent à une " réforme démocratique du système dans le cadre de l'islam » ainsi que la « remise en question du discours religieux wahhabite " (p.142). Commence, ainsi, une critique systématique du wahhabisme, non seulement de sa mise en action politique et sociale. Et, comme dans l'article sur la France, l'auteur présente une classification des différentes tendances du nouveau discours. Il en différencie quatre : la critique libérale, qui dénonce « au nom de principes se réclamant plus d'un humanisme universaliste que de l'islam à proprement parler les manifestations du wahhabisme » (p. 146); la critique islamique, "qui oppose la "modération" de l'islam aux "excès" du wahhabisme " (p.147); la critique salafiste, qui démontre «le paradoxe d'un wahhabisme tiraillé entre un idéal d'ijtihad affirmé et une pratique juridique figée dans un hanbalisme conservateur " (p.150); et la critique "wahhabite ", qui "rappelle la pluralité interne de la tradition wahhabite, et prône sa réforme de l'intérieur, en se proposant d'y redonner cours aux tendances inclusivistes face à la mainmise des exclusivistes " (p. 153). De la part de l'État, on observe des encouragements, bien que très prudents, envers ces courants, ce qui permet de parler des premiers tâtonnements d'une ouverture sous le regard bienveillant du roi.

Le contexte des discours islamiques en Palestine se présente d'une façon compliquée et multiple, le peuple palestinien étant éparpillé - géographiquement, politiquement, culturellement. " Nous voulons ici suggérer, dit l'auteure, que c'est l'impérative de la résistance, bien plus que "l'islam", qui explique le recul du discours laïc au profit du 
discours religieux » (p.166). Elle explique en détail l'absence de société civile ainsi que d'espace public qui ne permet pas (comme le dit Jürgen Habermas) le développement d'un débat intellectuel. Donc, "c'est dans l'espace fictif du "débat", que la société palestinienne s'est inventé d'Oslo I à Intifada II » (p. 171).

14 Le champ intellectuel islamique en Égypte est monopolisé depuis vingt ans par les mêmes figures vieillissantes qui sont, aujourd'hui, doublées par les «intermédiaires culturels islamistes» (p.180) dont l'enjeu «n'est alors plus de construire une alternative islamique globale à l'Occident " (id.) Cette mouvance est née de la crise des grands récits islamiques et implique, aussi, une critique de la politique totalitaire des Frères musulmans qui désiraient incorporer un islam totalisant dans une organisation totalisante. En considérant ceci comme une imitation de l'état postcolonial, ces intermédiaires culturels islamistes demandent et travaillent au changement culturel et normatif dans la société. Dans ce contexte, "c'est moins une nouvelle orientation idéologique clairement marquée qui se constitue que la reconnaissance de fait du mélange des genres et de l'extraversion conceptuelle» (p. 187). Ce qui donne une nouvelle compréhension aux concepts comme jihād et $d a^{\prime} w a$, dont le premier peut désormais signifier tout « du hacktivisme à la société civile en passant par la réalisation de soi et la pensée positive» (p.188), dont le seconde peut désormais signifier «le travail, le sérieux et la rigueur » (p.191). C'est une prise de distance avec «la posture identitaire propre au grand récit islamiste », clairement visible chez Amr Khaled, ce nouveau prêcheur qui veut "réunir à nouveau "la religion et la vie" sans laisser la première s'hypertrophier au point d'étouffer la seconde» (p. 195). Ainsi, l'islam est tenu hors la politique, ce qui rend plus flexible et plus ouverte la politique parce qu'on ne cherche plus les éléments de la modernité politique dans les textes islamiques. C'est un tournant significatif, un nouveau commencement.

Ce dossier est une source formidable pour les développements les plus récents des discours internationaux musulmans, bien qu'aucun des auteurs n'ose indiquer, statistiquement, l'importance ou l'impact politique et social de ces voix nouvelles. 